Relations industrielles

Industrial Relations

\title{
Barling, Julian, Clive Fullagar, and E. Kevin Kelloway. The Union and its Members : A Psychological Approach
}

\section{Renaud Paquet}

Volume 48, numéro 3, 1993

URI : https://id.erudit.org/iderudit/050887ar

DOI : https://doi.org/10.7202/050887ar

Aller au sommaire du numéro

Éditeur(s)

Département des relations industrielles de l'Université Laval

ISSN

0034-379X (imprimé)

1703-8138 (numérique)

Découvrir la revue

Citer ce compte rendu

Paquet, R. (1993). Compte rendu de [Barling, Julian, Clive Fullagar, and E. Kevin Kelloway. The Union and its Members : A Psychological Approach]. Relations industrielles / Industrial Relations, 48(3), 588-590.

https://doi.org/10.7202/050887ar

Tous droits réservés @ Département des relations industrielles de l'Université Laval, 1993
Ce document est protégé par la loi sur le droit d'auteur. L’utilisation des services d'Érudit (y compris la reproduction) est assujettie à sa politique d'utilisation que vous pouvez consulter en ligne.

https://apropos.erudit.org/fr/usagers/politique-dutilisation/ 
on shop stewards, without an appraisal of its relevance to North American unions. The authors are also precluded from examining the links between higher level leadership, membership characteristics and participation, and union structure.

Another chapter deals with the breakdown in unionization, i.e. workers' decisions to quit unions or terminate union representation. A key finding is that perceived union instrumentality is useful in predicting collective and individual action against the union. The last review chapter examines the impact of unions on organization behaviors ranging from the setting of compensation levels to employee satisfaction and turnover.

A concluding chapter emphasizes the primary objective of the book - "to illustrate how a knowledge of organizational psychology and unions could be enhanced by an understanding of each other"' (p. 192). The authors review some major findings, for example that there are important distinctions between specific and general union attitudes, and latter raise conceptual and methodological concerns for future studies, for example that demographic variables play only very limited roles in explanations of the unionization process. Despite their enthusiasm for behavioral research, the authors warn that a psychological approach may be important and necessary for an understanding of the unionization process, but by itself it is insufficient and should be complemented by legal and economic analyses in interdisciplinary studies.

I expect that readers will be distracted by some errors that should have been caught by an experienced copy editor at Oxford University Press. For example, I am sure that more than " 45,519 workers in the United States were involved in certification votes" from 1973 to 1978 (p. 152) and that without further explanation most readers will have difficulty deciphering the statement that the Canadian public generally approves of the overall functions of unions but disapproves of the unions' behavior. There is also some misplaced punctuation, unnecessary repetition of citations, and awkward phrasing, e.g. "attitudes to unionism in general"' (p. 158). But these are all minor points. The fact remains that there is no other book like this. The authors' contribution goes well beyond that of a literature review or reference work; by identifying the gaps in our knowledge of the psychology of unionism they present a clear and comprehensive agenda for the growing number of researchers in the field. I anticipate that this volume will be widely read and updated frequently.

Gary N. Chaison

Clark University

The Union and Its Members: A Psychological Approach, by Julian Barling, Clive Fullagar, and Kevin Kelloway, New York, Oxford University Press, 1992, 251 p., ISBN 0-19-507336-3.

De l'avis même des auteurs, la psychologie organisationnelle ignore le rôle des syndicats à l'égard des comportements organisationnels. Parmi les ouvrages se réclamant de cette discipline, rares sont ceux qui se sont intéressés à l'étude du syndicalisme. Celui de Barling, Fullagar et Kelloway constitue un effort pour combler ce vide. Il expose de façon convaincante comment les modèles explicatifs et les résultats empiriques de recherches en psychologie peuvent contribuer à un élargissement des 
connaissances, tant à l'égard du fonctionnement des organisations que de celui du comportement des individus à l'intérieur des syndicats.

Comme l'ont fait Murray et Verge à partir du droit et Freeman et Medoff de l'économie, les auteurs mettent à contribution leur discipline pour analyser l'institution syndicale. Autant les deux premiers ouvrages sont essentiels pour comprendre les dimensions formelles et les impacts du syndicalisme, autant celui-ci nous permet de jeter un regard des plus pertinents à l'intérieur de cette boîte souvent laissée obscure par la recherche et de mieux saisir la relation entre le salarié et son syndicat. À cet égard, les auteurs présentent une analyse du processus de syndicalisation. Ce dernier comprend à la fois le développement initial de l'attitude syndicale, la prise de position lors du vote d'accréditation, l'engagement et la participation syndicale et la décision de maintenir l'adhésion syndicale.

Après avoir introduit leur ouvrage au premier chapitre, les auteurs traitent au chapitre suivant de la structure des organisations syndicales. Ce chapitre comprend un survol des principales recherches empiriques traitant des déterminants des structures et des impacts de ces dernières sur l'efficacité syndicale, sur la démocratie syndicale ainsi que sur la relation entre le salarié et son syndicat. Il semblerait que plus la structure est bureaucratique et complexe, moins les salariés peuvent exercer de l'influence sur le processus décisionnel. Quant à l'impact sur la négociation collective, les résultats des recherches ne sont pas concluants.

Ce chapitre manque à notre avis de cohésion et il est difficile d'y suivre le fil conducteur. Les cinq chapitres qui suivent constituent le cœur de l'ouvrage. Ils portent sur les différentes étapes du processus de syndicalisation. Dans un premier temps, la littérature sur les déterminants de la syndicalisation est passée en revue. Les différentes variables y sont présentées sous les catégories suivantes : déterminants démographiques, caractéristiques personnelles, attitude à l'égard du travail, déterminants de niveau micro et de niveau macro. Après avoir présenté quelques modèles conceptuels, les auteurs concluent que les déterminants les plus stables pour prédire la syndicalisation sont : la faible satisfaction au travail, l'attitude syndicale et la perception de l'instrumentalité syndicale. En fait, les salariés se syndiqueraient, non pas pour des motifs idéologiques ou politiques, mais plutôt pour ce qu'ils peuvent en tirer en vue de la satisfaction de leurs besoins reliés au travail.

Les deuxième et troisième dimensions du processus de syndicalisation proposées par les auteurs ont trait à l'engagement (chapitre IV) et à la participation (chapitre V) syndicales. Les auteurs ajoutent les résultats de leurs recherches à ceux de Gordon et ses collègues (1980) pour présenter un modèle d'engagement syndical. Dans ce modèle, l'engagement comporte quatre dimensions : la loyauté syndicale, l'adhésion aux objectifs défendus par le syndicat, la propension à travailler pour le syndicat et celle à assumer ses responsabilités de membre. Alors que les deux premières dimensions sont voisines du concept de l'attitude syndicale, les deux dernières se rapprochent de celui du comportement (participation) syndical. Les déterminants de l'engagement syndical sont sensiblement les mêmes que ceux de la syndicalisation initiale si ce n'est que les caractéristiques structurelles du syndicat prennent plus d'importance. Quant aux variables explicatives de la participation syndicale, elles y sont aussi présentées. La faible 
satisfaction au travail, l'instrumentalité syndicale perçue et l'attitude syndicale sont les variables retenues par les auteurs.

Au chapitre VI, il est question du délégué syndical, de son rôle et de son influence ainsi que des facteurs déterminants de la propension à occuper une telle charge. Les différentes recherches sur le sujet y sont révisées. Le délégué syndical est ici présenté comme le lien essentiel entre les salariés et leur syndicat. La façon dont il s'acquitte de ses fonctions et le style de leadership qui le caractérise ne sera pas sans influencer l'attitude des salariés à l'égard de leur syndicat. Les différentes variables démographiques sont peu utiles pour prédire qui deviendra délégué syndical. Par contre, les convictions syndicales, le profil idéologique et le souci du bien-être des collègues de travail sont souvent associés au fait d'occuper une charge syndicale.

Après avoir étudiè la phase initiale de la syndicalisation et celles de l'engagement et de la participation, les auteurs, au chapitre VII, poursuivent avec la phase finale (pour certains) que constitue la désaffiliation syndicale. La littérature sur la désaffiliation syndicale est beaucoup plus limitée que celle sur l'affiliation. Toutefois, il s'en dégage que les facteurs explicatifs des deux phénomènes sont sensiblement les mêmes à l'exception du degré de satisfaction au travail qui est reliée à la syndicalisation mais ne l'est pas à la désyndicalisation. En effet, le salarié limitera son évaluation à la dimension instrumentale du syndicat lorsqu'il sera appelé à se prononcer sur le maintien de l'affiliation. Avant la syndicalisation, l'évaluation de l'instrumentalité était plus spéculative alors qu'à cette étape elle est de nature factuelle.

Le dernier chapitre traite de l'effet syndical sur les comportements organisationnels. Après avoir présenté les impacts du syndicalisme (selon la littérature) à partir des résultats directs de la négociation collective (salaires, avantages, sécurité d'emploi...), les auteurs se tournent vers les résultats indirects. Alors que pour le premier type d'enjeux les résultats sont évidents, il est difficile de dégager un consensus de la littérature à l'égard de l'impact du syndicalisme sur des enjeux comme l'absentéisme, la productivité et la performance, la satisfaction au travail et le stress.

L'ouvrage de Barling, Fullagar et Kelloway constitue une contribution dans la littérature scientifique traitant du syndicalisme. Les auteurs ont su y présenter, dans un tout des plus cohérent, les résultats des multiples recherches pertinentes. L'originalité de l'ouvrage découle avant tout de l'approche privilégiée (psychologie) et du fil conducteur unique de l'ouvrage, soit le processus de syndicalisation. Chaque chapitre comprend une revue de la littérature, une tentative d'intégration des connaissances et des propositions quant aux pistes futures de recherche. Il s'agit selon nous d'un ouvrage essentiel pour l'étudiant ou le chercheur qui désire améliorer sa compréhension de la relation « personnelle » qui existe entre le salarié et le syndicalisme.

Renaud PaqueT

Université du Québec à Hull 